Last year, President Clinton made clear the urgent need for an effective HIV vaccine, and suggested that it should be possible to create one within ten years. The authors agree. Although recent progress should not be underestimated, many obstacles remain - some technical, others not. Here, Dennis Burton (Scripps Research Institute) and John Moore (Aaron Diamond AIDS Research Center) outline their views on the state of development of an HIV vaccine and the areas in which more effort should be focused.

\title{
Why do we not have an HIV vaccine and how can we make one?
}

The most fundamental question to ask about an HIV vaccine is: 'What evidence exists that protection against disease after exposure to HIV is possible?' The best evi-

dence for successful protection against a virulent primate lentivirus such as HIV is that monkeys are almost always protected against challenge with pathogenic SIV $_{\text {mac }}$ after vaccination with an attenuated (nef-deleted) SIV $_{\text {mac }}$ strain $^{1}$. Whether this particular observation can be directly or indirectly exploited for the development of an HIV vaccine for humans is an important issue, to which we return below.

There is evidence that neutralizing antibodies can protect against HIV infection in certain animal models ${ }^{2}$. However, the antibody concentrations required are high and the specificities of effective antibodies are uncommon. The overwhelming majority of antibodies to envelope immunogens produced during natural infection or vaccination are probably elicited and affinity matured against envelope proteins in conformations different from that present on the virion surface. Most of these antibodies then bind weakly or not at all to virions and are functionally ineffective. Very few antibodies capable of binding to and neutralizing a broad spectrum of representative primary viruses have been generated during natural infection ${ }^{3}$, none yet by vaccination. A fundamental problem is the poor immunogenicity of the mature oligomeric glycoprotein complex that constitutes the surface spikes of HIV. The limited accessibility of the critical neutralizing antibody epitopes on the virion also means that antibody binding is relatively inefficient ${ }^{2,4}$. Even the best HIV-neutralizing antibodies are at least an order of magnitude less effective than the best neutralizing antibodies to polio virus, for example. Although it will not be easy to induce antibodies of the right quality in sufficient quantity by vaccination, this must be an important goal.

Although there is no direct evidence that cytotoxic T lymphocytes (CTL) protect against challenge with HIV or its simian counterparts, there is a wealth of data showing a correlation between the appearance of CTL activity and the containment of viral infection $^{5-7}$. For example, the early CTL response in one HIV-infected person was focused on a highly immunodominant epitope of gp 160, leading to the rapid elimination of the initially dominant strain (transmitted virus) ${ }^{8}$. The selection pressure of the CTL response was even sufficient to drive the emergence of a CTL escape mutant. That CTL (but not antibody) responses can be detected in certain very highly exposed, yet HIV-uninfected, African prostitutes also argues for the protective effect of cellular immunity; these individuals may have been naturally vaccinated against HIV by a mechanism that is unknown, but obviously important to determine $e^{9}$. Thus, the induction of a strong CTL response is also on most peoples' vaccine wish list.

Overall, we believe that both antibody and CTL responses will need to be induced for an HIV vaccine to be effective. The view that both arms of the immune system are required is also sup- ported by studies in mice challenged with retroviruses and other RNA viruses ${ }^{10-12}$. By reducing the infectivity of the initial viral inoculum, neutralizing antibodies pronse time to mature and clear those cells which do become infected. CD4 ${ }^{+} \mathrm{T}$-cell help is essential for both humoral and cellular immune responses to work effectively ${ }^{10}$; interference with T-helper cell functions is probably one of the ways by which HIV prevents the immune system from clearing the virus during natural infection ${ }^{13}$. Vaccine priming of T-cell help may buy the immune system time in the critical, initial race between HIV replication and immune-mediated viral clearance $^{10}$. Whether other, less well characterized innate or immune responses might also be advantageous to an HIV vaccine is unclear, but they are certainly worth exploring.

There has been a surprising paradigm shift in HIV vaccine research during the past few years: It used to be the perception that making a significant, vaccine-induced antibody response to HIV was easy, whereas it would be extremely difficult to make an effective CTL response. Now, however, CTL responses can be induced fairly efficiently by some immunogens (at least in mice), but a meaningful neutralizing antibody response remains elusive. This problem must be overcome, urgently.

\section{The current strategies}

Historically, most successful vaccines have been made of attenuated virus or killed virus. However, both of these approaches are problematic for HIV. Although attenuated viruses are likely to be quite effective, there are genuine safety concerns, as emphasized by recent reports ${ }^{14}$ of morbidity in monkeys after vaccination with attenuated (nef-deleted) SIV $_{\text {mac. }}$. Whether this would necessarily be so with HIV in humans is uncertain; trials are necessary to prove safety, but there is a reluctance to approve trials because of the perceived safety concerns-an unfortunate Catch 22 . Whether additional attenuation of SIV (and by analogy HIV-1) would retain its protective potential while further reducing virulence is an important area of investigation and one where there are already encouraging signs of progress.

Irrespective of the continued debate about human studies, research on attenuated $\operatorname{SIV}_{\text {mac }}$ in monkeys remains essential because of the power of the induced protection. Understanding its cause is of the utmost importance for HIV vaccine development, for the mechanism is still sadly unclear. Critical roles have been alternatively proposed and refuted for both humoral (neutralizing antibodies) and cellular (CTL) immunity ${ }^{15}$. A 'viral interference' model has also been invoked, in which the attenuated virus occupies all the available 'niches' and thereby prevents a virulent virus from gaining a foothold ${ }^{16}$. Cross-protection studies involving SHIVs ${ }^{17}$, and other studies, indicate that neutralizing antibodies are probably not all that important for protection by attenuated viruses, which is not to say that antibodies are unimportant for vaccine design in general. If cellular immunity 
indeed dominates the protection achieved by attenuated viruses, a central, perplexing riddle is: How does the vaccine strain prevent an incoming virus from becoming established, yet not eliminate itself? Likewise, humans naturally infected with a nef-deleted virus have not eradicated this virus from their bodies ${ }^{18}$. Why not?

The major problem associated with a killed HIV vaccine involves inactivating the virus without losing or destroying the fragile envelope glycoproteins. Because killed vaccines work by inducing neutralizing antibodies, there is little to be gained by using an immunogen that lacks the antigens which induce them, either prophylactically or post-infection. A further substantial obstacle is the sheer difficulty of producing useful quantities of primary isolate virions. These problems, although

very significant, are at least visible for attack.

Subunit envelope vaccines using recombinant forms of the HIV envelope glycoproteins (particularly gp120) are safe, but have been disappointing ${ }^{19}$. To date, these proteins (or derived peptides) have not elicited significant neutralizing antibody responses to representative primary viruse ${ }^{20}$, almost certainly because epitope exposure differs between recombinant proteins and the mature oligomer found on the virus ${ }^{2,4}$. Nor do soluble proteins elicit strong $\mathrm{CD}^{+} \mathrm{CTL}$ responses. Current attempts to make recombinant gp120s induce CTLs by the use of experimental adjuvants, run the risk of denaturing the protein and thereby destroying any chance of evoking a relevant antibody response ${ }^{21}$. It would seem prudent to focus on the potential strength of the recombinant protein in generating antibodies and leave CTL induction to other vaccine constructs. For recombinant envelope glycoproteins to be able to make a meaningful contribution to a vaccine cocktail, however, much needs to be done to increase the immunogenicity of the present generation of proteins, both by changing their antigenic structure and by developing better methods of presenting them exogenously. Ideally, a useful recombinant protein should improve on the qualities of the mature oligomer, as found on the virion surface, although this does not necessarily mean that oligomeric proteins per se are the answer to the problem. Much more effort is needed to understand the HIV envelope glycoproteins and to exploit them as immunogens.

Live recombinant vectors (such as avipox, canarypox or vaccinia) that include HIV antigens, and naked DNA vectors which also express these proteins, are important approaches still at a relatively early stage of what one hopes will be a highly successful evolution ${ }^{22,23}$. DNA vectors, which include multiple HIV antigens, seem more promising than ones which focus on a single antigen (for example, gp160). Expression of the encoded antigens in humans and monkeys also needs to be optimized by the evaluation of different promoters and the improvement of delivery systems; this is an especially important function of non-human primate models. However, neither live viral vectors nor DNA has so far induced worthwhile antibody responses to the HIV envelope glycoproteins-their strength lies in their ability to stimulate cellular immune responses, particularly CTLs. Additional antibody responses can be induced by 'boosting' the live vector- or DNA-primed immune system with soluble recombinant gp120. However, these

\section{IMAGE UNAVAILABLE FOR COPYRIGHT REASONS}

Vaccines generally aim to elicit neutralizing antibodies and cytotoxic T lymphocytes (CTLS). fragment) coating the surface of rhinovirus. CTLs kill infected cells. Fig. $1 b$ shows stages in the of a target cell.

are the same proteins that have performed poorly as sole immunogens and there is little evidence that they are working any better as part of a more complex vaccine-indeed, there are few reasons why they should. The CTL-inductive powers of live virus vectors and DNA need to be supplemented by a greatly improved series of antibody-inducing recombinant proteins.

\section{Why is an HIV vaccine so difficult?}

Many hypotheses have been proposed to explain the difficulties in generating a vaccine to HIV when vaccines against other viruses have been successful. These include a rapid replication rate for HIV, viral variation and the importance for immunity of the $\mathrm{CD}^{+}$target cell that HIV infects and destroys. Whereas such factors are clearly significant for the development of disease, it is not readily apparent why alone they should create insurmountable obstacles to a vaccine. The replication rate of HIV is not remarkable compared to other viruses for which vaccines exist. Influenza is highly variable, but vaccines against individual influenza strains are readily developed whereas protecting against molecular clones of HIV (SIV) has proven very difficult. Measles virus infects cells of the immune system and produces immunosuppression but a vaccine can still prevent measles infection.

We believe the most important distinguishing features of HIV are the nature of the virus envelope and the ability of a retrovirus to integrate into host DNA. As noted above, the properties of the mature envelope glycoprotein complex on the viral surface render the virus 'stealth-like' with respect to the humoral immune system. It is extremely difficult to present the envelope glycoproteins in a way that stimulates a significant antibody response or memory. The integration capability of HIV means that, unless sterilizing immunity is achieved, some of the infecting virus will remain invisible to humoral and cellular immune responses. Activation will release infectious virus until sufficient diversity is generated to nullify the efficacy of vaccine-induced immunity.

\section{The great action debate}

There is an ongoing debate between those who favor emphasizing large trials of candidate HIV vaccines in humans to determine efficacy, and those who believe more basic knowledge must be attained before large trials would be warranted. The empirical approach has worked in the past for other pathogens and so naturally attracts much support. However this approach has ap- 
peared unlikely to generate an HIV vaccine due to some of the complexities discussed above. Some argue that there is no harm in testing many different vaccine candidates, however weak the underlying scientific justification or preliminary data. We do not accept this argument, except at the Phase I level where we partially endorse it (see below). First, funding for HIV vaccine research is not unlimited: resources allocated to large trials of inadequate vaccines could be better spent in developing a stronger vaccine. Second, the patient cohorts needed for Phase III trials are a precious resource that should be called upon with great care. Third, there is a social and political price to be paid for failed vaccines: losing the support of the public for a health measure as important as an HIV vaccine would be a disaster. Whatever the external pressure 'to be seen to be doing something,' large vaccine trials must be based on good science and a reasonable chance of achieving something worthwhile, and not just on the availability of an immunogen.

An empirical approach can teach but it is not true that one always learns from failure. An efficacy trial of a gp120 subunit vaccine based on a lab. strain (as is pending approval by the FDA) will not tell us that no gp120-based recombinant protein could ever work, only that the particular product is inadequate, something which is already clear enough from Phase I/II trials ${ }^{19,24}$. There is a disturbing tendency in HIV vaccine research to dress up failure as success, or to design the conditions of the experiment to improve the likelihood of an apparent success. For example, primary viruses have a spectrum of antibody neutralization sensitivity. There are even some primary viruses which are almost as easy to neutralize as the lab. strains (for example, IIIB and MN), that so misled the vaccine field for many years, but they are not representative. Choosing neutralization-sensitive viruses to give apparently positive results has obvious short-term appeal, but in the long term, it is a practice that will not facilitate the development of an effective HIV vaccine. Similarly, some groups focus on avirulent and/or readily neutralized viruses. These viruses can be valuable for eliminating a concept from further considerationfailure to protect against, for example, SHIV-IIIB in macaques would be a most discouraging sign-but success against such viruses should not be overemphasized in the way it so often is. Arguably the most misleading of the easy protection models is the HIV-1 SF-2 challenged chimpanzee. Here HIV-1 replication is so weak and protection so easy to achieve that any positive conclusions drawn about the prospects of vaccinating humans against virulent HIV-1 strains should attract considerable skepticism.

Despite the problems associated with the poor humoral immune responses to the live recombinant vector-prime/gp120boost vaccines, there is considerable pressure to move these vaccines rapidly from limited Phase II trials into Phase III trials. Is this justified? Results from the first generation of vacciniaprime/gp120-boost vaccines have been disappointing in terms of the breadth and duration of the CTL response ${ }^{25}$. Because induction of a CTL response is the theoretical strength of the prime-boost approach, major improvements to the first generation of immunogens are clearly required prior to conducting Phase III trials. An additional point to consider is that a CTLbased vaccine is unlikely to induce sterilizing immunity. It is then difficult to judge vaccine efficacy in trials carried out in developed countries because ethical re- quirements will surely mandate that any individuals infected during a vaccine trial will be immediately treated with antiretroviral drugs, as noted recently by Barry Bloom ${ }^{26}$. Whatever decision is taken about efficacy trials of the present prime-boost vaccines, it would be prudent to assume that these vaccines, in their current form, will fail and to plan accordingly.

\section{Government-directed and investigator-initiated research}

Should the AIDS vaccine effort in the United States be strongly directed by the National Institutes of Health $(\mathrm{NIH})$ and other federal agencies, or carried out via a myriad of investigator-initiated projects? The answer is both. HIV vaccine development requires the kind of leaps forward classically made by individuals with insight and drive who work in intramural and extramural research laboratories. Yet, it could also greatly benefit from direction that introduced standardization. For example, simian models have a crucial role to play in HIV vaccine development ${ }^{1}$, but there are too many individual models. The number and variety of different challenge stocks, animal species and experimental protocols strongly mitigate against comparisons of different concepts tested in different research laboratories. And no single laboratory has the resources to conduct satisfactory experimentation on more than one or two concepts. How can anyone possibly judge whether a DNA vaccine tested in a rhesus macaque challenged with SIVmac-251 is superior to a gp120-subunit tested in a nemestrina macaque challenged with HIV-2 or to a prime-boost vaccine in cynomolgus monkeys challenged with SIV delta70? This morass needs urgent resolution by simplification and consolidation worldwide. This was accomplished because of financial necessity in Europe, with benefits that are now clear.

It is a general premise that the less a challenge stock replicates in a host, the easier it is to protect against it. A rational strategy for animal models would involve the convergence of the field around two well-tested models at opposite ends of the pathogenicity-protection spectrum, and perhaps one in the middle of the spectrum. The 'easy' model can be used to eliminate concepts, as noted above, the 'hard' one to explore the limits of their potential. These 'approved' models would be used for strictly comparative evaluations of vaccine concepts, to refine and improve their performance along with similar studies in humans, where challenge experiments are obviously not possible.

Thus we urge a more centralized direction to human and animal trials aimed at HIV vaccine development. This needs to go hand-in-hand with investigator-initiated research. To quote Gustav Nossal ${ }^{27}$ : '... in the early years of the new millennium, ... the third golden age of immunology (will help create) a panoply of new vaccines'. However, some areas of vaccine development are so expensive and complex that they are beyond the financial
Table 1 Status of current HIV-1 vaccine strategies

\begin{tabular}{|c|c|c|c|c|}
\hline \multirow[t]{2}{*}{$\begin{array}{l}\text { Candidate } \\
\text { Vaccine }\end{array}$} & \multirow[t]{2}{*}{$\begin{array}{l}\text { safety } \\
\text { concerns }\end{array}$} & \multicolumn{2}{|c|}{$\begin{array}{c}\text { potency of elicited immune } \\
\text { response }\end{array}$} & \multirow{2}{*}{$\begin{array}{l}\text { other undefined } \\
\text { protective } \\
\text { mechanisms }\end{array}$} \\
\hline & & Neutralizing antibody & dy CTL. & \\
\hline Attenuated virus & Yes & Poor & merging evidence & Some evidence \\
\hline Killed virus & Limited & Poor & Negligible & No evidence \\
\hline Envelope subunits & None & Poor & Negligible & No evidence \\
\hline $\begin{array}{l}\text { Vaccinia or avipox } \\
\text { prime/boost }\end{array}$ & None & Poor & Weak & No evidence \\
\hline DNA prime/boost & Limited & Poor & Weak & No evidence \\
\hline
\end{tabular}


and administrative scope of individual investigators. It is here that central direction is most needed. In the USA, this should be provided by the NIH and in particular by the NIH's AIDS Vaccine Research Committee working closely with the directors of those institutes most involved in HIV vaccine research.

\section{The role of biopharmaceutical companies}

The experience of biopharmaceutical companies in the HIV vaccine area has not been a happy one. Creation of an HIV vaccine has been seen too much as product development, too little as experimental sciencè. Companies and their academic collaborators have been reluctant to acknowledge the failure of their favored HIV vaccine-there seem to be many trials, few errors. Instead, interpretive loopholes are ruthlessly exploited to breathe life into a corpse, as is now occurring with the subunit gp120 vaccines. To reverse this trend, we urge the NIH and its principal advisors to play a primary role in selecting immunogens for comparative evaluation in multiple Phase I trials. The financial responsibility for making and testing a range of immunogens for these early clinical trials could be carried by the NIH under the contract mechanism; this might properly be viewed as experimentation, rather than product development per se. Comparative immunogenicity studies in humans could be supported by challenge experiments using analogous SHIVs in monkeys, if the concept under evaluation is suitable for this model. The emphasis of Phase I human trials must be squarely on the rejection of poorly performing concepts before too many resources are devoted to them. Clearly, this is alien to corporate practice, and quite beyond the financial capability of smaller companies, which is why alternative funding mechanisms must be developed. Only the more promising immunogens should be advanced to Phase II and beyond, and it is at this stage that corporate experience in vaccine development will be essential. Ultimately, it is the larger biopharmaceutical companies that will produce an HIV vaccine on a large scale and bring it to the public. Their continued involvement is crucial.

\section{Conclusions}

Why do we not have an HIV vaccine? We attribute this to the 'stealth-like' qualities of the viral envelope glycoproteins with respect to the humoral immune system, combined with the ability of the virus to integrate into host cells to emerge later, diversify and escape CTL control. How can we make an HIV vaccine? Understanding the mechanisms for the protection afforded by attenuated viruses is vital. Both CTL and antibody responses are likely to be necessary. Significant progress has been made in eliciting CTL responses, although much remains to be done in primates. To induce good antibody responses, enhancing the immunogenicity and antigenicity of the HIV envelope are crucial steps. Animal models need rationalizing, but remain of central importance. Human clinical trials must be guided by the wealth of knowledge accumulating from basic studies of immunology and virology, with the immediate emphasis on experimentation at the Phase I level rather than on product development. The NIH should commission immunogens designed under the direction of the AIDS Vaccine Research Committee. Judgment, and not absolute certainty, will be necessary to evaluate Phase I (and II) studies; this can also be provided by the same committee. The need for independent evaluation of trials is highlighted by the very different conclusions reached from the same clinical trial of a gp120 subunit vaccine $\mathrm{e}^{19,24,28}$. Central direction by the AIDS Vaccine Research Committee, working with senior figures in the institutes it advises, could hasten the pace of vaccine research in the United States. The powers of this committee should be strengthened in the coming months and its performance must only be judged over a period measured in years. There will be no 'quick fix' to the HIV vaccine problem: none should be expected.

1. Desrosiers, R.C. Non-human primate models for AIDS vaccines. AIDS 9 (Suppl. A), S137-S141 (1995).

2. Burton, D.R. A vaccine for HIV type 1: the antibody perspective. Proc. Natl. Acad. Sci. USA 94, 10018-10023 (1997).

3. Trkola, A. et al. Cross-clade neutralization of primary isolates of human immunodeficiency virus type 1 by human monoclonal antibodies and tetrameric CD4-lgG. I. Virol. 69, 11, 6609-6617 (1995).

4. Moore, J.P. \& Ho, D.D. HIV-1 neutralization: the consequences of viral adaptation to growth on transformed T cells. AIDS 9 (suppl A), S117-\$136 (1995).

5. Rowland-Jones, S., Tan, R. \& McMichael, A. The role of cellular immunity in protection against HIV infection. Adv. Immunol. 65, 448-455 (1997).

6. Jassoy, C. \& Walker, B.D. HIV-1-specific cytotoxic T lymphocytes and the control of HIV-1 replication. Springer Sem. Immunopathol. 18, 341-354 (1997).

7. Oldstone, M.B. A. HIV versus cytotoxic T lymphocytes: the war being lost. N. Eng. J. Med. 337, 1306-1308 (1997).

8. Borrow, P. et al. Antiviral pressure exerted by HIV-1-specific cytotoxic T lymphocytes (CTLs) during primary infection demonstrated by rapid selection of CTL escape virus. Nature Med. 3, 205-211 (1997).

9. Rowland-Jones, S.L. \& MCMichael, A. Immune responses in HIV-exposed seronegatives: have they repelled the virus? Curr. Opin. Immunol. 7, 448-455 (1995).

10. Hasenkrug, K.J. \& Chesebro, B. Immunity to retroviral infection: the Friend virus model. Proc. Natl. Acad. Sci. USA 94, 7811-7816 (1997).

11. Baldridge, J.R., McGraw, T.S., Paoletti, A. \& Buchmeier, M.). Antibody prevents the establishment of persistent arenavirus infection in synergy with endogenous $T$ cells. 1. Virol. 71, 755-758 (1997).

12. Planz, O. et al. A critical role for neutralizing-antibody-producing B cells, CD4+ $T$ cells, and interferons in persistent and acute infections of mice with lymphocyte choriomeningitis virus: implications for adoptive immunotherapy of virus carriers. Proc. Natl. Acad. Sci. USA 94, 6874-6879 (1997).

13. Rosenberg, E.S. et al. Vigorous HIV-1-specific CD4+T cell response associated with control of viremia. Science 278, 1447-1450 (1997).

14. Ezzell, C. The monkey's got AIDS: what now for live AIDS vaccines? Journal of NIH Research 9, 21-22 (1997).

15. Stott, E.J. \& Schild, G.C. Strategies for AIDS vaccines. /. Antimicro. Chemother. 37 Suppl. B, 185-198 (1996).

16. Sharpe, S.A., Whatmore, A.M., Hall, G.A. \& Cranage, M.P. Macaques infected with attenuated simian immunodeficiency virus resist superinfection with virulence-revertant virus. J. Gen. Virol. 78, 1923-1927 (1997).

17. Shibata, R., Siemon, C., Czajak, S.C., Desrosiers, R. C. \& Martin, M.A. Live, attenuated simian immunodeficiency virus vaccines elicit potent resistance against a challenge with a human immunodeficiency virus type 1 chimeric virus. J. Virol. 71, 8141-8148 (1997)

18. Deacon, N.J. et al. Genomic structure of an attenuated quasi species of HIV-1 from a blood transfusion donor and recipients. Science 270, 988-991 (1995).

19. Connor, R.I. et al. Immunological and virological analyses of persons infected by human immunodeficieny virus type 1 while participating in trials of recombinant gp120 subunit vaccines. f. Virol. 72, 1552-1576 (1998).

20. Haynes, B.F. HIV vaccines: where we are and where we are going. Lancet 348 , 933-937 (1996)

21. VanCott, T.C. et al. Antibodies with specificity to native gp 120 and neutralization activity against primary human immunodeficiency virus type 1 isolates elicited by immunization with oligomeric gp1 20. /. Virol. 71, 4319-4330 (1997).

22. Excler, J.-L. \& Plotkin, S. The prime-boost concept applied to HIV preventative vaccines. AIDS 11, $5127-5137$ (1997)

23. Liu, M.A. . Nature Med. (1998). See this issue [Insert page \#s].

24. Graham, B.S. et al. Analysis of intercurrent human immunodeficiency virus type 1 infections in phase I and II trials of current AIDS vaccines. J. Infect. Dis. 177, 310-319 (1998).

25. Corey, L. et al. Cytotoxic $T$ cell and neutralizing antibody responses to human immunodeficiency virus type 1 envelope with a combination vaccine regimen. I Infect. Dis. 177, 301-309 (1998).

26. Bloom, B.R. The highest attainable standard: ethical issues in AIDS vaccines. Science 279, 186-188 (1998).

27. Nossal, G. Host immunobiology and vaccine development. The Lancet 350 , 1316-1319 (1997).

28. Berman, P.W. et al. Genetic and immunologic characterization of viruses infecting MN-rgp120-vaccinated volunteers. J. Infect. Dis. 176, 384-397 (1997).

${ }^{1}$ Departments of Immunology and Molecular Biology, The Scripps Research Institute, 10550 North Torrey Pines Road, La Jolla, California 92037, USA email: burton@scripps.edu

${ }^{2}$ The Aaron Diamond AIDS Research Center, The Rockefeller University, 455 First Ave, New York, New York 10016, USA

email:jmoore@adarc.org 